\title{
Intestinal obstruction: predictor of poor prognosis in colorectal carcinoma?
}

\author{
Mohd Azri Mohd Suan, Wei Leong Tan, Shahrul Aiman Soelar, Ibtisam Ismail, \\ Muhammad Radzi Abu Hassan \\ Clinical Research Center, Sultanah Bahiyah Hospital, Kedah, Malaysia
}

OBJECTIVES: The goal of this study was to assess the relationship between intestinal obstruction and the prognosis of colorectal carcinoma.

METHODS: Data pertaining to 4,501 colorectal carcinoma patients were extracted from the national colorectal registry and analysed. Survival analysis was performed using the Kaplan-Meier method. The log-rank test was used to compare the survival rate between patients with intestinal obstruction and those without intestinal obstruction. The p-values $<0.05$ were considered to indicate statistical significance. Simple Cox proportional hazards regression analysis was used to estimate the crude hazard ratio of mortality from colorectal cancer.

RESULTS: Intestinal obstruction was reported in more than $13 \%$ of patients. The 3 -year survival rate after treatment was $48.3 \%$ (95\% confidence interval [CI], 43.9 to 52.8 ) for patients with intestinal obstruction $(n=593)$ and $54.9 \%(95 \%$ CI, 53.1 to 56.6$)$ for patients without intestinal obstruction $(n=3,908)$. The 5 -year survival rate for patients with intestinal obstruction was $37.3 \%(95 \% \mathrm{CI}, 31.9$ to 42.8$)$, which was lower than that of patients without intestinal obstruction $(45.6 \%$; $95 \%$ CI, 43.5 to 47.7). After adjusting the hazard ratio for other prognostic variables, intestinal obstruction had a statistically significant negative correlation with the survival rate of colorectal cancer patients, with an adjusted hazard ratio of $1.22(\mathrm{p}=0.008)$.

CONCLUSIONS: The presence of intestinal obstruction is associated with a lower survival rate among colorectal cancer patients.

KEY WORDS: Carcinoma, Colorectal neoplasm, Intestinal obstruction, Prognosis

\section{INTRODUCTION}

Colorectal carcinoma is one of the most widespread malignancies worldwide. In 2012, colorectal carcinoma became the third most common cancer globally, with nearly 1.4 million new cases diagnosed [1]. The age-standardized rate for both sexes

\section{Correspondence: Mohd Azri Mohd Suan}

Clinical Research Center, Sultanah Bahiyah Hospital, 05640 Alor Setar, Kedah, Malaysia

Tel: +60-134395159, Fax: +60-47407373, E-mail: irzah96@yahoo.com

Received: Jan 5, 2015, Accepted: Mar 30, 2015, Published: Mar 30, 2015

This article is available from: http://e-epih.org/

(C) 2015, Korean Society of Epidemiology

(C) This is an open-access article distributed under the terms of the Creative Commons Attribution License (http://creativecommons.org/licenses/by/3.0/), which permits unrestricted use, distribution, and reproduction in any medium, provided the original work is properly cited. differs from country to country; the age-standardized rate for males was 41.5 to 61.6 per 100,000 , and the age-standardized rate for females was 25.4 to 35.8 per 100,000 [1]. The incidence rate was found to be higher in developed countries than in developing countries for both sexes [1]. Colorectal carcinoma was most frequently reported cancer in males and the second most frequently reported cancer in females in peninsular Malaysia according to the 2006 report of the Malaysian National Cancer Registry [2].

Many factors affecting the prognosis of colorectal carcinoma have been studied. According to Park et al. [3], colorectal carcinoma is a complex clinical problem, and high risks of mortality were associated with advanced-stage tumours, elevated preoperative serum carcinoembryonic antigen, rectal cancer, and ulcero-infiltrative tumours. In the present study, we assessed the prognosis of colorectal carcinoma patients with and without in- 
testinal obstruction.

Malignant large bowel obstruction is a clinical presentation associated with a significant likelihood of colorectal carcinoma. Markogiannakis et al. [4] found that colorectal carcinoma was one of the top three causes of intestinal obstruction (13.4\%) among 150 patients who presented with intestinal obstruction. The effects of obstruction on the large bowel included ischemia (16.6\%), necrosis (16.6\%), and perforation (11.1\%) [4].

In order to address the relationship between intestinal obstruction and the prognosis of colorectal carcinoma, we examined data from a large multicentre national colorectal registry. Our main goal was to compare the survival duration of colorectal cancer patients with and without intestinal obstruction. Other variables that might affect the survival duration of patients with colorectal carcinoma were also extracted from the registry, including age, sex, ethnicity, education level, the presence of diabetes mellitus, and cancer staging.

\section{MATERIALSAND METHODS}

A cross sectional study was conducted using secondary data from the National Cancer Patient Registry Colorectal Cancer. This registry was established in October 2007, with current coverage of 34 hospitals across all parts of Malaysia. The registry coordinating centre is based at the Clinical Research Centre, Sultanah Bahiyah Hospital, Alor Setar, Kedah, Malaysia. This registry is registered with the National Medical Research Registry (NMRR-07-49-242) with the aim of systematically collecting data on all aspects of colorectal carcinoma relevant to its prevention and treatment.

This study used data from 4,501 patients who were diagnosed with histologically proven colorectal carcinoma between 2008 and 2013. All data were entered online into the registry database by 34 hospitals, which are known as site data providers. The registry coordinating centre tracked data returns and prompted the site data provider if there was any problem with data collection. For the purposes of this study, data were locked on April 30, 2014 and sent from the database server to the coordinating centre for data cleaning. The data included demographic information (e.g., age, sex, ethnicity, current status of patient), diabetes status, and cancer staging upon diagnosis. The current status of registered patients (whether still alive or deceased) was ascertained by cross-checking the data with the National Registration Department, Ministry of Home Affairs.

Data analyses were performed with SPSS version 20.0 (IBM Corp., Armonk, NY, USA). The frequencies of each demographic characteristic, the presence of intestinal obstruction, cancer staging upon clinical presentation, and the presence of co-morbidities were obtained. No imputation was carried out for miss- ing values. The 3-year and 5-year survival analyses were performed using the Kaplan-Meier method, estimating the median survival time and survival rate. The log-rank test was used to compare the survival rate between patients with intestinal obstruction and patients without intestinal obstruction. The p-values $<0.05$ were considered to indicate statistical significance [5]. Simple Cox proportional hazards regression analysis was used to determine the crude hazard ratio of mortality from colorectal cancer associated with intestinal obstruction, using patients without intestinal obstruction as a reference group. The hazard ratio associated with intestinal obstruction, using patients without intestinal obstruction as a reference group, was then adjusted by age, sex, ethnicity, diabetes mellitus, and cancer staging, using multiple Cox proportional hazards regression analysis (using the 'enter' method).

\section{RESULTS}

\section{Patient characteristics}

The intestinal obstruction status of all 4,501 patients from the registry database was recorded; $86.8 \%(n=3,908)$ did not have intestinal obstruction and $13.2 \%(n=593)$ did have intestinal obstruction. Most of the colorectal carcinoma patients with intestinal obstruction were in the 60-69-year-old age group (29.7\%), followed by the 70 -year-old or more age group (27.2\%). The mean age for our patients was 61.6 years old (standard deviation= 12.72 years).

The sex distribution showed a preponderance of male patients with intestinal obstruction $(57 \% ; n=338)$ compared to females $(42.5 \% ; n=252)$. The Malay ethnicity was the majority $(44.5 \%)$ in our cohort, followed closely by Chinese $(35.9 \%)$, others $(15.3 \%)$, and Indian (4.2\%). Of the 4,501 colorectal carcinoma patients, $2,490(55.3 \%)$ were deceased at the time of analysis. The group of deceased patients included 297 patients with intestinal obstruction. Information about the demographic characteristics of patients is provided in detail in Table 1.

The Kaplan-Meier estimates of the 3-year survival rate after treatment (Table 2, Figure 1) were $48.3 \%$ (95\% confidence interval [CI], 43.9 to 52.8 ) for patients with intestinal obstruction $(\mathrm{n}=593)$ and $54.9 \%$ (95\% CI, 53.1 to 56.6$)$ for patients without intestinal obstruction $(\mathrm{n}=3,908)$. The 5 -year survival rate for patients with intestinal obstruction was 37.3\% (95\% CI, 31.9 to 42.8 ), which was lower than that of patients without intestinal obstruction $(45.6 \%$; 95\% CI, 43.5 to 47.7$)$.The logrank test was used to compare the survival rates of patients with intestinal obstruction and patients without intestinal obstruction, and a statistically significant difference was found $(p=0.005)$.

The implications of intestinal obstruction were further analysed using Cox proportional hazards regression analysis in or- 
Table 1. Demographic characteristics of colorectal carcinoma patients with and without intestinal obstruction, 2008-2013

\begin{tabular}{|c|c|c|}
\hline Demographic characteristics & $\begin{array}{l}\text { Patients with intes- } \\
\text { tinal obstruction }\end{array}$ & $\begin{array}{l}\text { Patients without in- } \\
\text { testinal obstruction }\end{array}$ \\
\hline \multicolumn{3}{|l|}{ Age (yr) } \\
\hline$\leq 19$ & $6(1.0)$ & $5(0.1)$ \\
\hline $20-29$ & $15(2.5)$ & $49(1.3)$ \\
\hline $30-39$ & $27(4.5)$ & $150(3.8)$ \\
\hline $40-49$ & $70(11.8)$ & $452(11.6)$ \\
\hline $50-59$ & $138(23.3)$ & $954(24.4)$ \\
\hline $60-69$ & $176(29.7)$ & $1,187(30.4)$ \\
\hline$\geq 70$ & $161(27.2)$ & $1,068(27.3)$ \\
\hline Missing & - & $43(1.1)$ \\
\hline Total & $593(100.0)$ & $3,908(100.0)$ \\
\hline \multicolumn{3}{|l|}{ Sex } \\
\hline Male & $338(57.0)$ & $2,180(55.8)$ \\
\hline Female & $252(42.5)$ & $1,681(43.0)$ \\
\hline Missing & $3(0.5)$ & $47(1.2)$ \\
\hline Total & $593(100)$ & $3,908(100.0)$ \\
\hline \multicolumn{3}{|l|}{ Ethnicity } \\
\hline Malay & $264(44.5)$ & $1,658(42.4)$ \\
\hline Chinese & $213(35.9)$ & $1,601(40.9)$ \\
\hline Indian & $25(4.2)$ & $234(6.0)$ \\
\hline Others & $91(15.3)$ & $393(10.1)$ \\
\hline Missing & - & $22(0.6)$ \\
\hline Total & $593(100.0)$ & $3,908(100.0)$ \\
\hline \multicolumn{3}{|l|}{ Diabetes mellitus } \\
\hline Yes & $430(72.5)$ & $2,680(68.6)$ \\
\hline No & $143(24.1)$ & $862(22.0)$ \\
\hline Missing & $20(3.4)$ & $366(9.4)$ \\
\hline Total & $593(100)$ & $3,908(100.0)$ \\
\hline \multicolumn{3}{|l|}{ Stage upon diagnosis } \\
\hline | & $31(5.2)$ & 347 (8.9) \\
\hline$\|$ & $132(22.3)$ & $815(20.9)$ \\
\hline III & $196(33.1)$ & $1,032(26.4)$ \\
\hline IV & $126(21.2)$ & $783(20.0)$ \\
\hline Missing & $108(18.2)$ & $931(23.8)$ \\
\hline Total & $593(100.0)$ & $3,908(100.0)$ \\
\hline \multicolumn{3}{|c|}{ Current status (as of April 30, 2014) } \\
\hline Dead & $297(50.1)$ & $2,193(56.1)$ \\
\hline Alive & $296(49.9)$ & $1,715(43.9)$ \\
\hline Missing & - & - \\
\hline Total & $593(100.0)$ & $3,908(100.0)$ \\
\hline
\end{tabular}

Values are presented as number (\%).

der to determine hazard ratios for the mortality of colorectal carcinoma patients (Table 3). The analysis revealed that intestinal obstruction had a significant negative effect on colorectal carcinoma survival $(p=0.005)$. Specifically, the risk of colorectal carcinoma death was 1.19 times higher for patients with intestinal obstruction compared to those without intestinal obstruction. After adjusting the hazard ratio for other prognostic variables, namely, age, sex, ethnicity, diabetes mellitus, and cancer staging, intestinal obstruction among colorectal cancer patients was found to be a statistically significant factor for poor survival, with an adjusted hazard ratio of $1.22(\mathrm{p}=0.008)$.
Table 2. Median survival time and survival rate of colorectal carcinoma patients with and without intestinal obstruction from 2008-2013

\begin{tabular}{|c|c|c|c|c|c|}
\hline \multirow{2}{*}{ Variables } & \multirow{2}{*}{$\begin{array}{l}\text { Median } \\
(95 \% \mathrm{Cl})\end{array}$} & \multicolumn{2}{|c|}{ Survival rate (\%) } & \multirow{2}{*}{$\chi^{2}(d f)$} & \multirow{2}{*}{$p$-value ${ }^{1}$} \\
\hline & & $3 y r$ & $5 \mathrm{yr}$ & & \\
\hline Intestinal obstruction & & & & $7.88(1)$ & 0.005 \\
\hline Yes & $2.84(2.29,3.40)$ & 48.3 & 37.3 & & \\
\hline No & $4.02(3.61,4.43)$ & 54.9 & 45.6 & & \\
\hline
\end{tabular}

Survival analysis using the Kaplan-Meier method.

Median, median survival time in years; $\mathrm{Cl}$, confidence interval; $\chi^{2}$, chi-squared; $\mathrm{df}$, degrees of freedom.

${ }^{1}$ Log-rank test.

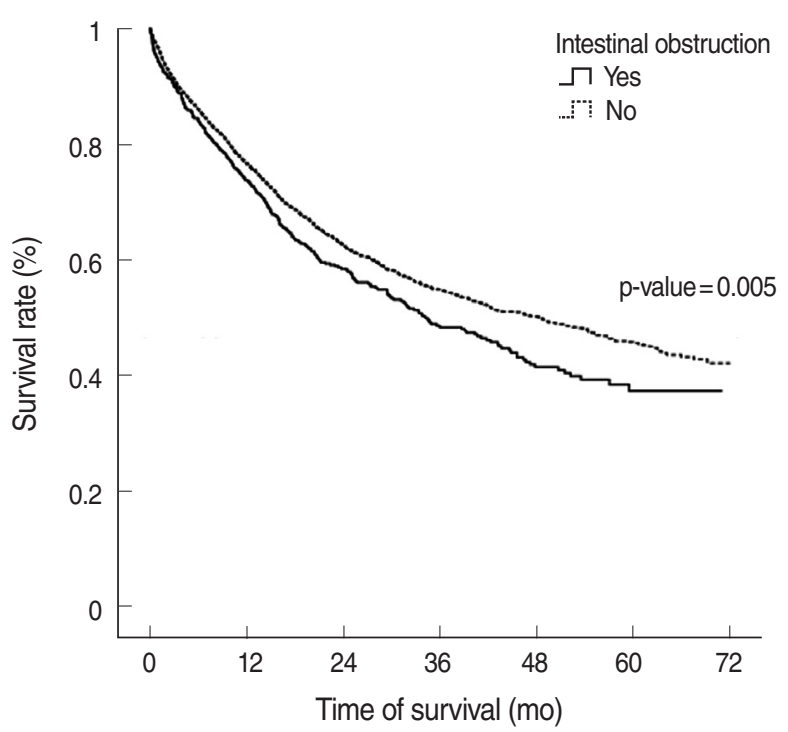

Figure 1. Kaplan-Meier curve of the survival of colorectal carcinoma patients with and without intestinal obstruction.

Table 3. Prognosis of colorectal carcinoma with or without intestinal obstruction

\begin{tabular}{lcccc}
\hline Variables & $\begin{array}{c}\text { Crude } \mathrm{HR} \\
(95 \% \mathrm{Cl})\end{array}$ & p-value $^{1}$ & $\begin{array}{c}\text { Adjusted HR } \\
(95 \% \mathrm{Cl})\end{array}$ & $\mathrm{p}$-value \\
\hline Intestinal obstruction & 0.005 & & 0.008 \\
Yes & $1.19(1.06,1.35)$ & & $1.22(1.06,1.42)$ & \\
No & 1.00 (reference) & & 1.00 (reference) & \\
\hline
\end{tabular}

$\mathrm{HR}$, hazard ratio; $\mathrm{Cl}$, confidence interval.

${ }^{1}$ Simple Cox proportional hazards regression analysis.

'Multiple Cox proportional hazards regression analysis using the 'enter' method, adjusted by age, gender, ethnicity, diabetes mellitus, and cancer staging.

\section{DISCUSSION}

Many prognostic factors have been identified in connection with colorectal cancer, with the goal of estimating the chance of recovery or recurrence. Previously, other researchers have reported several adverse prognostic factors in patients with colo- 
rectal cancer including age, tumour differentiation, gross tumour morphology, primary tumour resection, and lymph node metastasis [6-8].

The current study focused on intestinal obstruction as an important factor affecting the prognosis of colorectal cancer. More than $13 \%$ of patients $(n=593)$ were reported to have intestinal obstruction and were included in the analysis. This figure was consistent with the findings of other studies, which have reported an incidence of intestinal obstruction of 10 to $29 \%$ in colorectal cancer patients [9-11]. Although the number of patients who presented with intestinal obstruction is not large, the presence of this condition has been shown to be associated with negative outcomes. Obstruction of the intestinal lumen develops slowly and often remains partial. The gastrointestinal symptoms caused by the obstructed intestine usually depend on the site of obstruction. A left-side lesion usually presents as an alteration in bowel habits and the passage of blood, while lesions on the right side usually present as anaemia and dull aching pain [12].

The current study determined that the patients without intestinal obstruction in our cohort had a longer median survival time (4.02 years) than those with intestinal obstruction (2.84 years). Patients with intestinal obstruction had a 3-year survival rate of $48.3 \%$, compared to a 3 -year survival rate of $54.9 \%$ in patients without intestinal obstruction. The 5-year survival rate of patients with intestinal obstruction was $37.3 \%$, which was lower than that of patients without intestinal obstruction (45.6\%). Furthermore, the adjusted hazard ratio for death from colorectal carcinoma was 1.22 times higher in patients with intestinal obstruction, compared to those without intestinal obstruction.

The shorter survival duration among colorectal cancer patients who presented with intestinal obstruction is similar to the findings of other investigators. Yuan et al. [6] conducted a univariate analysis of prognostic factors for patients with colorectal cancer and found that the 3-year and 5-year survival rates for patients with intestinal obstruction were only 39 and $35 \%$, respectively. However, further multivariate analysis found no association between intestinal obstruction status and survival. Rasool et al. [13] conducted a study on patients with a history of intestinal obstruction due to colorectal carcinoma over a period of 13 years in India. Only 13 patients survived after a period of five years, with a survival rate of $23.12 \%$. The lower survival rate in that study, compared to our findings, may have been due to the small number of patients in their cohort. The prognosis was even worse among patients who presented with intestinal obstruction and advanced-stage cancer. Several studies have demonstrated that the survival period for such patients ranges from a few weeks to several months [14-16]. For instance, Chen et al. [16] studied the survival duration of patients with stage IV colorectal cancer complicated with bowel obstruction. Among 381 patients who had been diagnosed with stage IV disease, 295 patients had intestinal obstruction. The mean survival time was 49.4 and 37.2 months for the non-obstruction and obstruction groups, respectively.

There are several possible reasons for poor survival outcomes among colorectal cancer patients with intestinal obstruction. It has been postulated that patients with intestinal obstruction already present at an advanced stage of cancer. This situation leads to poor nutritional dynamics and later triggers other biochemical imbalances that increase the mortality of colorectal cancer $[14,17]$. Moreover, patients with intestinal obstruction require more extensive debulking surgery. Since the majority of patients in our cohort were elderly (more than 50 years old), post-surgery mortality and morbidity were more frequent in this group due to their poor health status and poor nutritional intake. ScottConner et al. [18] also reported increased rates of surgical complications in patients of advanced age. The presence of intestinal obstruction may also lead to micro-metastasis to the nearby mesenteric lymph nodes. This type of micro-metastasis in the lymph nodes is not easily detected. In fact, Gusterson et al. [19] estimated that only $1 \%$ of pathologists are able to identify a small metastatic focus of cancer that metastases to the regional lymph nodes. Such failure to detect micro-metastases may lead to under-staging the severity of the tumour and under-treatment of the patient, which may contribute to the poor survival rate.

This study had several limitations. Firstly, this analysis was based on data obtained from the National Cancer Patient Registry Colorectal Cancer, which involves numerous hospitals and site data providers nationwide. The presence of incomplete data was one of the main issues that occurred during data collection. Hence, continuous training on data collection and data entry for all source data providers may help minimize this problem. Secondly, the authors were unable to determine an adjusted hazard ratio taking other variables into account, such as sites of obstruction and surgical intervention, as has been done in other studies $[14,15,20]$. This was due to limitations in the data available in the registry. The coordinating centre for the registry has added additional variables (including the above-mentioned variables) in the new registry form to strengthen data capture for colorectal cancer. These reforms will perhaps provide better data for future analysis.

In conclusion, colorectal cancer patients without intestinal obstruction showed a tendency to live longer than those with intestinal obstruction, and the difference between the two groups was significant. Therefore, patients should be advised to seek medical treatment as soon as clinical symptoms appear, in order to improve the outcomes of colorectal cancer. This can be achieved by improving public awareness on the prevention and early treatment of colorectal cancer through colorectal cancer screening. 


\section{ACKNOWLEDGEMENTS}

The authors would like to thank the Director General of Health, Malaysia, for his permission to publish this paper. Special thanks to all participating hospitals who work tirelessly to ensure that the data in the National Colorectal Cancer Registry is updated. We also thank all the individuals who directly or indirectly have contributed to the completion of this study.

\section{CONFLICT OF INTEREST}

The authors have no conflicts of interest to declare for this study.

\section{REFERENCES}

1. International Agency for Research on Cancer. GLOBOCAN 2012: estimated cancer incidence, mortality and prevalence worldwide in 2012. [cited 2015 Apr 30]. Available from: http://globocan.iarc.fr/ Default.aspx.

2. National Cancer Registry, Ministry of Health Malaysia. Malaysian cancer statistics-data and figure peninsular Malaysia 2006 [cited 2015 Apr 30]. Available from: http://www.moh.gov.my/images/gallery/ Report/Cancer/MalaysiaCancerStatistics_2006.pdf.

3. Park YJ, Park KJ, Park JG, Lee KU, Choe KJ, Kim JP. Prognostic factors in 2230 Korean colorectal cancer patients: analysis of consecutively operated cases. World J Surg 1999;23:721-726.

4. Markogiannakis H, Messaris E, Dardamanis D, Pararas N, Tzertzemelis D, Giannopoulos P, et al. Acute mechanical bowel obstruction: clinical presentation, etiology, management and outcome. World J Gastroenterol 2007;13:432-437.

5. Lang TA, Secic M. How to report statistics in medicine: annotated guidelines for authors, editors, and reviewers. 2nd ed. Philadelphia: American College of Physicians; 2006, p. 45-50.

6. Yuan Y, Li MD, Hu HG, Dong CX, Chen JQ, Li XF, et al. Prognostic and survival analysis of 837 Chinese colorectal cancer patients. World J Gastroenterol 2013;19:2650-2659.

7. Zhang S, Gao F, Luo J, Yang J. Prognostic factors in survival of colorectal cancer patients with synchronous liver metastasis. Colorectal
Dis 2010;12:754-761.

8. Compton CC. Colorectal carcinoma: diagnostic, prognostic, and molecular features. Mod Pathol 2003;16:376-388.

9. Tuca A, Guell E, Martinez-Losada E, Codorniu N. Malignant bowel obstruction in advanced cancer patients: epidemiology, management, and factors influencing spontaneous resolution. Cancer Manag Res 2012;4:159-169.

10. Chan KK, Dassanayake B, Deen R, Wickramarachchi RE, Kumarage SK, Samita S, et al. Young patients with colorectal cancer have poor survival in the first twenty months after operation and predictable survival in the medium and long-term: analysis of survival and prognostic markers. World J Surg Oncol 2010;8:82.

11. Zucchetti F, Negro F, Matera D, Bolognini S, Mafucci S. Colorectal cancer: obstruction is an independent negative prognostic factor after radical resection. Ann Ital Chir 2002;73:421-425.

12. Libutti SK, Tepper J, Saltz LB, Rustgi A. Cancer of the rectum. In: DeVita VT, Hellman S, Rosenberg SA, editors. Cancer, principles and practice of oncology. 7th ed. Philadelphia: Lippincott Williams \& Wilkins; 2005, p. 1110.

13. Rasool A, Bari S, Rashid S, Wani A, Wani R, Peer G. Outcome of patients with acute intestinal obstruction due to colorectal carcinoma. Internet J Surg 2008;20:1.

14. Ripamonti C, Twycross R, Baines M, Bozzetti F, Capri S, De Conno $\mathrm{F}$, et al. Clinical-practice recommendations for the management of bowel obstruction in patients with end-stage cancer. Support Care Cancer 2001;9:223-233.

15. Krouse RS. Surgical management of malignant bowel obstruction. Surg Oncol Clin N Am 2004;13:479-490.

16. Chen W, Tan XP, Ye JW, Liu Q, Zeng Q, Wang L, et al. Effect of bowel obstruction on stage IV colorectal cancer. Mol Clin Oncol 2014;2:308-312.

17. Gallardo-Valverde JM, Calañas-Continente A, Baena-Delgado E, Zurera-Tendero L, Vázquez-Martínez C, Membrives-Obrero A, et al. Obstruction in patients with colorectal cancer increases morbidity and mortality in association with altered nutritional status. Nutr Cancer 2005;53:169-176.

18. Scott-Conner CE, Scher KS. Implications of emergency operations on the colon. Am J Surg 1987;153:535-540.

19. Occult axillary lymph-node micrometastases in breast cancer. Lancet 1990;336:434-435.

20. Medina-Franco H, García-Alvarez MN, Ortiz-López LJ, Cuairán JZ. Predictors of adverse surgical outcome in the management of malignant bowel obstruction. Rev Invest Clin 2008;60:212-216. 